Théologiques

Théologiques

\title{
Retrouver la dimension cosmologique de la théologie chrétienne
}

\section{Jean Proulx}

Volume 9, numéro 1, printemps 2001

Les cosmologies

URI : https://id.erudit.org/iderudit/005689ar

DOI : https://doi.org/10.7202/005689ar

Aller au sommaire du numéro

Éditeur(s)

Faculté de théologie de l'Université de Montréal

ISSN

1188-7109 (imprimé)

1492-1413 (numérique)

Découvrir la revue

Citer cet article

Proulx, J. (2001). Retrouver la dimension cosmologique de la théologie chrétienne. Théologiques, 9(1), 49-70. https://doi.org/10.7202/005689ar
Résumé de l'article

Cet article montre qu'un aspect essentiel du combat spirituel de la théologie chrétienne, aujourd'hui, est de retrouver sa dimension cosmologique. Puisant à ses propres sources, la théologie peut accueillir de façon critique la cosmologie scientifique contemporaine (la " nouvelle physique ") et ses prolongements et enracinements ontologiques." Récapitulant " ainsi science et philosophie, la théologie les conduit vers leur propre dépassement ; et, grâce à elles, elle chemine elle-même vers sa vérité la plus riche, puisqu'elle est amenée à relire les représentations et les contenus essentiels de la foi : Dieu, la création, le Christ, l'Église, la liturgie et l'espérance. 


\title{
Retrouver la dimension cosmologique de la théologie chrétienne
}

\author{
Jean ProulX \\ Département de Sc. Religieuses \\ Université du Québec à Montréal
}

La théologie doit satisfaire deux besoins inséparables : établir le noyau de vérité du message chrétien, d'une part, et réinterpréter cette vérité pour chaque génération, d'autre part. Elle vit donc une tension à la fois ardue et féconde entre la fidélité au message essentiel de la révélation et la situation conceptuelle de l'époque. C'est en ce sens que Tillich parle, de la méthode de corrélation entre le message révélé et la situation historique ${ }^{1}$, et Siegwalt, de la méthode de coordination entre la démarche prophétique de la foi et la démarche sapientielle de la raison $^{2}$. La raison s'exprime dans la science et aussi dans la philosophie qui, elle-même, se doit d'accueillir de façon critique les implications philosophiques de la science. À son tour, la théologie est appelée à accueillir lucidement les analyses et les questions mises de l'avant par la science et la philosophie. Sinon, il y a rupture, compartimentage et dualisme dans la maison du savoir : La théologie risque alors d'emprunter le chemin qui la conduit vers une sorte de supranaturalisme désincarné, et les sciences et la philosophie, pour leur part, monde désenchanté, privé de sens et vidé de la présence de Dieu.

La théologie chrétienne occidentale a été trop peu ouverte à la cosmologie scientifique contemporaine et à sa portée philosophique et théologique. On peut parler d'une cécité de la théologie occidentale

1. Paul Tillich, Théologie systématique. L'être et Dieu. Paris, Planète, 1970, p. 19-29.

2. Gérard SIEGWALT, Dogmatique pour la catholicité évangélique. Tome III._L'affirmation de la foi. 1. Cosmologie théologique: Sciences et philosophie de la nature. Paris, Cerf, 1996, p. 9-31. 
dominante à l'égard du cosmos ${ }^{3}$. La théologie a été longtemps en effet anthropocentrique (centrée sur le salut personnel de l'homme) et acosmique (repliée loin de la cosmologie). Elle a semblé avoir capitulé devant la cosmologie scientifique, au lieu de la « récapituler ». Cela peut expliquer, pour une part, que nombre de grands physiciens contemporains, conscients de la portée philosophique et théologique de "la nouvelle physique », se soient plutôt tournés vers un dialogue avec la pensée mystique orientale. Pourtant, il y a, dans la nouvelle physique et la vision globale sur laquelle elle s'ouvre, une sollicitation actuelle que la théologie chrétienne peut accueillir.

La théologie chrétienne, puisant à ses propres sources et pratiquant la méthode de corrélation ou de coordination, peut en effet récapituler la cosmologie scientifique et ses prolongements ou ses enracinements philosophiques, c'est-à-dire les accueillir de façon critique et les conduire à leur accomplissement théologique. Il lui faut cependant pratiquer un dialogue lucide avec la nouvelle physique : discerner et retenir ce qui mérite de l'être selon son point de vue et, sans impérialisme, montrer comment on peut comprendre le cosmos de façon théonome ou comme création de Dieu ; accepter également la relecture de la foi qu'appelle cette écoute consciente de la cosmologie contemporaine. Il y va du combat spirituel de la raison : pour leur vérité, cosmologie scientifique et cosmologie ontologique sont appelées à s'ouvrir à la théologie; et, pour sa propre vérité, la théologie doit s'ouvrir à son tour à la science et à la philosophie ${ }^{4}$. Et ce, sans aucun dogmatisme, dans un contexte de partenariat et dans la conscience que la vérité, finalement, est une.

\section{La nouvelle physique et son prolongement ontologique ${ }^{5}$}

Ce que l'on appelle la nouvelle physique, qui naît principalement de la pensée quantique et de la théorie de la relativité au début du $\mathrm{XX}^{\mathrm{e}}$ siècle, conduit à la remise en question de la représentation de l'univers sur laquelle la science reposait et, conséquemment, de la place que

3. Ibid., p.152-159.

4. Ibid., p. 9.

5. J'ai développé ce thème dans un livre récent. Voir Jean ProulX, $\mathrm{La}$ chorégraphie divine. Montréal, Fides, 1999, 165 p. 
l'être humain y tient et même de l'image de la divinité qui peut en ressortir. À l'encontre de la pensée mécaniste, déterministe et fragmentaire de la physique des siècles précédents, la physique du $\mathrm{XX}^{\mathrm{e}}$ siècle jette sur le cosmos, comme nous le rappellerons brièvement, un regard organiciste, évolutionniste et holistique.

\subsection{Le cosmos comme Énergie créatrice}

Pour la nouvelle physique (la cosmologie scientifique actuelle), le cosmos est un océan d'énergie. C'est l'énergie qui danse et vibre dans la matière, selon les structures qu'elle lui donne ou les formes qu'elle lui imprime $^{6}$. Si l'on peut dire que l'énergie se matérialise, on peut également affirmer que la masse est énergie ${ }^{7}$. Dans le monde infiniment petit des particules élémentaires, cette énergie est fluide comme l'eau, et c'est son aspect ondulatoire ; mais elle s'échange et circule par petites quantités concentrées (les quanta), et c'est là son aspect corpusculaire. Dans l'infiniment grand, elle se déploie comme la danse incessante des galaxies, des étoiles et des planètes, selon le rythme des naissances, des morts et des renaissances.

L'énergie cosmique apparaît comme un champ de force primordial ${ }^{8}$. Ainsi, le cosmos, né du Grand Vide originel créateur de toutes les formes, se manifeste comme un domaine unifié dans lequel circulent les courants d'une énergie pure ${ }^{9}$; c'est un tout organique, une sorte de "Grand Vivant », exécutant une danse sans fin ${ }^{10}$. Il est

6. David BoHM, (et F.D. Peat). La danse de l'esprit, La Varenne SaintHilaire, Séveyrat, 1988, p. 153ss.

7. Gary ZuKAV, La danse des éléments, Paris, Robert Laffont, 1982, p. 52, 138.

8. Albert EINSTEIN, (et Léopold INFELD), L'évolution des idée en physique. Des premiers concepts aux théories de la relativité et des quanta, Paris, Petite Bibliothèque Payot, \# 47, 1963, p. 117ss ; ZuKAV, 1982, p. 212 ss ; BOHM, 1988 , p. 23 ss.

9. Michel CASSÉ, Du vide et de la création, Paris, Odile Jacob, 1995, p.79 ss et 161 ss ; Fritjof CAPRA, Le Tao de la physique. Paris, Tchou, 1979, p. 211 ss ; Rupert SHELDRAKE, La mémoire de l'univers, Paris, Du Rocher, 1988, p. 107ss ; Robert LINSSEN, L'univers, corps d'un seul vivant, Montréal, Libre Expression, 1990, p. 51.

10. Fritjof CAPra, Le Tao de la physique. Paris, Tchou, 1979, p. 229ss ; Gary ZuKav, La danse des éléments, Paris, Robert Laffont, 1982, passim. 
traversé par le " holomouvement ", cet élan créateur fondamental, ce courant de fond, duquel tous les êtres émergent et en lequel ils s'immergent ${ }^{11}$. Le battement de cœur du Grand Vivant cosmique est ce mouvement de dépliement et d'impliement, de création et de destruction. Depuis un quinzaine de milliards d'années, le holomouvement cosmique s'est aussi présenté foncièrement comme un élan novateur ${ }^{12}$ qui, dans une sorte de spirale de la complexité au sein même d'un incommensurable mouvement d'expansion, fait apparaître des formes multiples et variées, de plus en plus complexes et de plus en plus conscientes ${ }^{13}$.

\subsection{L'intelligibilité de l'univers}

Le champ énergétique cosmique se révèle aussi comme un champ de signification ou de conscience universelle ${ }^{14}$. Et d'abord, le cosmos se présente comme un langage ${ }^{15}$. Quarks, protons, neutrons, électrons, atomes, molécules, si minimes soient-ils, expriment déjà une organisation intelligente de la nature. Ils ont un sens et portent même un message. Ils font que cet univers est une musique et non pas un simple bruit. Le langage de l'univers, affirmait Einstein, est compréhensible et intelligible. Il présente une harmonie à " déchiffrer ", car justement il s'agit d'une harmonie " chiffrée ".

Non seulement l'univers «parle-t-il », mais encore « il pense », affirment à divers degrés de nombreux savants, tels Hoyle, Eddington, Wheeler, Talbot, Charron, Bohm, Capra, Bateson et Sheldrake. Cet univers manifeste de l'intelligence; il semble traversé par une

11. David BoHm, (et F.D. Peat). La danse de l'esprit, La Varenne SaintHilaire, Séveyrat, 1988, p. 20-21 ; David BOHM, La plénitude de l'univers, Paris, Du Rocher, 1987, p. 159ss.

12. Renée WeBER, Dialogue avec des scientifiques et des sages, Paris, Du Rocher, 1996, p.155 (entrevue avec Bohm) et 285 (entrevue avec Prigogine); voir aussi David BoHM, La conscience et l'univers, Paris, Du Rocher, 1990 et il y a PRIgogine (et Isabelle STEngers). La nouvelle alliance, Paris, Gallimard, 1986, 439 p.

13. Hubert ReEves, Patience dans l'azur, Montréal, Québec Science Éditeur, 1983, 259p.; Hubert ReEves, L'heure de s'enivrer, Paris, Seuil, 1986 , p. 53ss.

14. Rénée WEBER, 1996, entrevues avec Bohm et Sheldrake.

15. Hubert REEVES, 1986, p. 54-55. 
logique interne, voire habité par un méga-logiciel ${ }^{16}$. Il joue, avec intelligence, à organiser la matière. Cet univers, pourtant mystérieux, est profondément intelligible. "Dieu ne joue pas aux dés ", disait Einstein. Il y a une harmonie inhérente à la nature et l'évolution cosmique témoigne du fait que l'énergie, la matière et le sens marchent ensemble ${ }^{17}$. Certains physiciens affirment même que l'univers "savait » qu'il cheminait, à travers la montée de l'organisation et de la complexité, vers l'avènement de l'homme et de la conscience réflexive : c'est ce qu'on nomme le principe anthropique. À vrai dire, le cosmos se déploie comme le grand ballet de l'information. Il y a en lui une primauté de l'information sur la matière. L'organisation du cosmos repose sur l'information : c'est elle qui donne un sens à l'énergie et c'est elle qui permet l'ascension de la matière vers la plus grande complexité $^{18}$. Les champs nucléaire, électromagnétique et gravitationnel doivent être vus comme des champs d'information : les ondes qui y circulent la transportent et la transmettent, dans une danse ininterrompue.

On peut même affirmer que, à la source et à l'origine de notre monde matériel, changeant et divers, il y a un ordre plus subtil et plus profond, implicite et caché19. À ce niveau, l'univers forme un seul tout continu, chaque partie est reliée à toutes les autres et elle se déplie dans le tout comme le tout se replie en elle. Là, se situe le champ créateur engendrant toutes les formes et le fondement de toute information et de tout ordre explicite. En somme, voilà quelque chose comme une proto-intelligence ou une conscience cosmique.

16. Rupert SheldRAKe, La mémoire de l'univers, Paris, Du Rocher, 1988, passim ; Jean E. Charon, Les lumières de l'invisible, Paris, Albin Michel, 1985, p. 29-135 ; Michael TALBOT, L'univers : Dieu ou hasard, Paris, J'ai lu, (New Age \#2677), 1989, p. 171ss ; Robert LINSSEN, L'univers, corps d'un seul vivant, Montréal, Libre Expression, 1990, p. 99ss.

17. BOHM, 1988, p. 93-152.

18. ReEves, 1983, p. 54 ; Michael TALBOt, L'Univers : Dieu ou hasard. Paris, J'ai lu (New Age \# 2677) 1989, p. 129.

19. BOHM, 1990, chap. 3, 4, 5 ; WEBER, 1996, (entrevues avec BOHM). 


\subsection{Les forces liantes de l'unique tissu cosmique}

Plusieurs physiciens parlent de l'univers comme d'un unique tissu sans couture $^{20}$. Comme l'espace et le temps, les fils de chaîne et les fils de trame du cosmos forment une étoffe indivisible. La réalité, pour la nouvelle physique, est une ; elle constitue une totalité insécable. Toutes les parties sont liées les unes aux autres ; il n'y a pas de parties isolées ; la fragmentation n'est pas fondamentale. Chaque partie n'existe que parce que toutes les autres existent. Le cosmos constitue un tout organique ininterrompu ; il est habité, à vrai dire, par une profonde unité à laquelle nous appartenons. Des physiciens, tels Shrödinger, Wheeler, Bohm, Capra ou Chew, désignent cet " unus mundus ", ce monde unifié comme un «Je » ou comme une sorte de Grand Vivant ${ }^{21}$.

La transparence des parties entre elles et de chacune avec le tout semble bien être une autre caractéristique fondamentale de cet univers unifié, formant un seul tout organique. Selon la nouvelle physique, tout s'interpénètre. Il y a une telle transparence universelle que chaque partie du grand tout cosmique ressemble à un prisme, dans lequel se reflètent les autres parties et le tout lui-même. Ainsi, le cosmos apparaît non seulement comme un vaste système de présences mutuelles entre les éléments et les entités, mais également comme l'omniprésence du tout à chacune de ses parties. Cet univers est une structure qui relie toutes choses, un champ unifié dans lequel baignent toutes réalités, un réseau d'interactions, un hologramme où chaque partie est dans le tout et le tout en chaque partie ${ }^{22}$.

Il n'y a pas à se surprendre de cela, puisque de grandes forces d'association sont à l'œuvre dans l'univers ${ }^{23}$. De l'infiniment petit à l'infiniment grand, ces forces de liaison soudent des structures à la fois autonomes et solidaires entre elles. C'est la danse des énergies nucléaire, électromagnétique et gravitationnelle. La première retient les quarks

20. Par exemple, CAPRA, 1979, p.133ss ; BOHM, $1988: 32-36$.

21. LINSSEN, 1990, p. 35ss et 59ss ; ZuKAV, 1982, p. 309.

22. Gregory BATESON, La nature et la pensée, Paris, Seuil, 1984, p.16-17; BoHm, 1988, p. 5, 176 ; CAPRA, 1979, p. 289ss ; Michael TALBOT, Mysticisme et physique nouvelle, Paris, Mercure de France, 1984, p. 55ss, 83 ss.

23. ReEves, 1983, p.173ss ; ReEves, 1998, p. 86ss ; Trinh Xuan ThuAN, La mélodie secrète, Paris, Fayard, 1988, p. 127ss. 
dans les protons et les neutrons et combine ces derniers pour constituer les noyaux atomiques. La deuxième est responsable de l'apparition des structures atomiques et moléculaires et joue un rôle fondamental dans la cohésion des cellules et des organismes. La troisième assure la constitution et l'unité des systèmes massifs que sont les planètes, les étoiles et les galaxies. C'est pourquoi il y a, partout dans l'univers, les traces d'une profonde "volonté » d'association. Cette "volonté » ou cette " intention ", se demandent plusieurs physiciens, serait-elle le fait d'une unique superforce, créatrice de tout ? Y aurait-il une seule superénergie traversant l'univers et liant toutes choses ? Y aurait-il un seul champ de force fondamental créant une unique chorégraphie cosmique ${ }^{24}$ ?

\subsection{L'ouverture sur le mystère de l'Être et de Dieu}

La vision holistique, dynamique et organique de l'univers qui émerge de la nouvelle physique parvient au seuil du mystère de l'Être. Quand, après la question "Pourquoi y a-t-il quelque chose plutôt que rien ? ", les physiciens sont amenés à se poser des questions comme "Pourquoi y a-t-il de la musique plutôt que du bruit dans l'univers ? ", "Pourquoi toute cette gratuité dans le cosmos ? ", "Pourquoi une si grande précision mathématique dans l'univers ?" et "L'univers savait-il dès le début de son évolution qu'il cheminait vers l'homme ou la conscience réflexive ? ", alors ils soulèvent de grandes questions concernant le sens même de ce cosmos, au sein duquel l'être humain est né. Ils ouvrent, pour ainsi dire, la cosmologie scientifique sur la cosmologie ontologique ${ }^{25}$.

En effet, la cosmologie ontologique ${ }^{26}$ pose les questions ultimes concernant l'univers : "Pourquoi ? (son origine, sa cause première) ",

24. CAPRA, 1979, p.215 ; John GRIBBIN, À la poursuite du Big Bang, Paris, Flammarion, (Champs \#270), 1991, p. 361 ; Stephen HAWKING, Une brève histoire du temps, Paris, Flammarion, (Champs \#238), 1989, p. 203 ; Trinh Xuan Thuan, Le chaos et l'harmonie, Paris, Fayard, 1998, p. 329-337.

25. REEVES 1983, p.148 ; THUAN 1988, p. 81, 285, 299ss, 339 ; voir aussi, par exemple, BOHM 1988, passim.

26. Les grands noms de cette cosmologie ontologique auxquels je me réfère dans La Chorégraphie divine sont ceux, entre autres, de Parménide, Héraclite, Platon, Aristote, Thomas d'Aquin, Spinoza, Hegel, Schelling, Heidegger, Maritain, Lavelle, Bergson, Whitehead. 
"Quoi ? (son essence, sa substance) ", "En vue de quoi (son sens, sa finalité ) ? " La cosmologie ontologie nous fait donc pénétrer dans le « mystère de l'Être 27 ", posant les questions inévitables de la cause première et de la finalité ultime du tout cosmique ${ }^{28}$. Son savoir est radical ou premier et son horizon est l'unité dialectique de la totalité. Son espace est celui de la profondeur de l'Être, intuitionnée par la pensée au sein du devenir, de la matérialité et de la multiplicité. Elle (cette cosmologie ontologique) aussi, elle est habitée par une foi : c'est la foi de la raison dans le sens et en son triomphe sur l'absurde ou la foi ontologique en l'intelligibilité du mystère de l'Être et en sa victoire sur le non-être. Elle aussi, elle apparaît comme la « fides quaerens intellectum ».

Avec sa démarche sapientielle coïncidant avec l'éveil de la pensée philosophique, la cosmologie ontologique peut et doit intégrer de façon critique l'apport actuel de la nouvelle physique. Cette intégration critique est, à proprement parler, une reprise réflexive ou une récapitulation des données et conclusions de la cosmologie scientifique. Des concepts scientifiques peuvent alors être subsumés ou sublimés ou transcendés dans des concepts ontologiques (et analogiques, c'est-à-dire à la fois semblables et différents). C'est ainsi, par exemple, que le concept d'Être peut inclure en le dépassant le concept d'Énergie créatrice, que le concept de Logos peut inclure en le dépassant celui d'intelligibilité cosmique et que le concept d'Amour ou d'Élan vers l'unité peut inclure en le dépassant celui de Force de liaison ou d'Énergie liante ${ }^{29}$. Dans la cosmologie ontologique, le cosmos peut être interprété comme étant essentiellement le mystère du pouvoir créateur de l'Être, intelligible parce qu'imprégné par la structure signifiante du Logos présent au cœur de l'Être, et poursuivant comme sa fin ultime son retour à l'unité originelle de l'Être.

C'est ainsi que, au sein de l'immanence du cosmos, la cosmologie ontologique pressent une dimension de transcendance. Son "goût de l'Être » s'ouvre sur une « diaphanie de Dieu ${ }^{30}$ ». Elle se fait ontothéolo-

27. Jacques MARITAIN, Distinguer pour unir ou les degrés du savoir, Paris, Desclée de Bouwer, 1932, p. 8ss.

28. SiegWALT, 2000, p. 17.

29. Cette démarche d'inclusion et de dépassement est mise en oeuvre dans La Chorégraphie divine.

30. Pierre TeILHARD de Chardin, Le milieu divin. Paris, Seuil, 1957, p.159. 
gie, pour emprunter l'expression de Heidegger. Elle a l'intuition que la chorégraphie cosmique peut apparaître comme chorégraphie divine. L'espace ontologique, espace de l'immanence cosmique, peut donc aussi se révéler comme espace théologique, espace de la transcendance divine. De même que la cosmologie scientifique (la nouvelle physique) demande à être reprise par la pensée dans une cosmologie ontologique (la philosophie de la nature), de même cette dernière requiert à son tour d'être récapitulée dans une théologie ouverte à sa dimension cosmologique.

\section{La nécessaire dimension cosmologique de la théologie}

La théologie chrétienne se doit de retrouver et de réaffirmer aujourd'hui sa dimension cosmologique. Son dialogue avec la cosmologie contemporaine apparaît comme incontournable. Assumant de façon critique et avec discernement les acquis de la nouvelle physique et ses prolongements ou ses enracinements métaphysiques, elle peut les mener à leur dépassement. En revanche, pour sa propre vérité, elle doit accepter la relecture de ses contenus essentiels, relecture que sollicite son dialogue avec la cosmologie contemporaine. C'est une telle relecture cosmologique de la théologie qui n'est que brièvement évoquée dans les pages qui suivent.

\subsection{Dieu : la puissance infinie de l'Être}

La vision supranaturaliste de Dieu a tendance à se le représenter comme étant au-dehors de sa création, de l'autre côté de l'univers ou au-dessus du monde : c'est comme si Dieu intervenait de l'extérieur dans le cosmos. Elle objective Dieu dans son au-delà, accentuant sa transcendance. Elle en fait un être, suprême sans doute, mais ce super-être différent apparaît pourtant encore comme un segment de la totalité de l'être ${ }^{31}$. Le théisme personnaliste doit pouvoir être à la fois inclus et dépassé dans une représentation de Dieu comme Être en soi, Pouvoir de l'être auquel tout le cosmos participe, Fondement transpersonnel de tout ce qui est, y compris des personnes. Dieu inclut ainsi la réalité per-

31. Tillich, 1970, (1) p. 153, 166 ; Tillich 1970, (3) p. 30ss ; Ibid. 1971, (vol.2) p. 6, 11 ; SiegWalt, 2000, p. 361 ; Jürgen Moltmann, Dieu dans la création, Paris, Cerf, (Cogitatio Fidei \#146), 1988, p.13, 27-28 ; Matthew Fox, (1) La grâce originelle, Montréal, Bellarmin, 1995, p. 111. 
sonnelle, par son caractère concret, et la transcende, par son caractère ultime. Le symbole de "personne » appliqué à Dieu est effectivement analogique et symbolique; il doit être complété par d'autres symboles transpersonnels. Et c'est là, à ce point précis, que le discours théologique sur Dieu rejoint et assume la richesse symbolique que porte l'héritage de la cosmologie ontologique, avec ses concepts d'Être, de Fondement, de Puissance, de Substance et de Cause, par exemple ${ }^{32}$; et c'est là également qu'il rend possible une relecture symbolique et "sublimée » des concepts d'Énergie créatrice, de Champ infini, de Vide créateur et de Force unique, véhiculés par la nouvelle physique.

La représentation de Dieu qui accueille de façon critique et récapitule les cosmologies scientifique et ontologique ne peut être ni un théisme transcendant ni un panthéisme immanentiste. On peut la définir comme panenthéisme : Dieu n'est pas un super-être à côté du tout ; Dieu n'est pas le tout ; mais Dieu est en tout et tout est en Dieu ${ }^{33}$. Dieu apparaît ainsi comme le Fondement créateur (fascinant) et l'abîme incommensurable (terrifiant) de tout le cosmos, comme la Substance (immanente) et la Cause (transcendante) de la totalité de l'être. Il est le Pouvoir d'être infini au cœur et au-delà de toute chose ; il est aussi le Vide sans forme, créateur de toutes les formes du cosmos, comme disent des mystiques de toutes les traditions spirituelles et nombre de physiciens contemporains.

Enfin, ce Dieu est un Dieu vivant ${ }^{34}$. Il est un et dynamique, comme le cosmos qui le révèle. Il y a en lui une dialectique : le Pouvoir d'être est

32. Tillich, 1970, (1) p. 95ss, 118ss, 149ss ; SiegWalt, 2000, p.54; Moltmann, 1988, p.119.

33. Fox 1995, (1) p. 112, 131 ; Tillich 1970, (1) p. 149ss ; MoltmanN 1988, p. 133.

34. Tillich 1970, (1) p.175-178; Paul Tillich, Systematic Theology Chicago, University of Chicago Press, 1971, vol.3, p.283 ; SIEGWALT 2000, p.222; Moltmann 1988, p.30-32 ; Douglas John Hall Être image de Dieu, Paris, Cerf, (Cogitatio Fidei \#213), 1998, p.185ss. L'hindouisme voit aussi Dieu comme un et trine : Dieu est Sat (Être), Chid (Conscience) et Ananda (Béatitude amoureuse); voir à ce sujet Sri AUROBINDO, La vie divine, tome 1 ; il y a aussi, dans l'hindouisme, une trinité mythologique : Brahman (créateur), Vishnu (conservateur) et Shiva (destructeur-recréateur ). L'Égypte ancienne a aussi sa trinité mythologique : Amon (Dieu caché), $\mathrm{Ra}$ (Dieu révélé) et Ptha (le corps de Dieu). Voir à ce sujet DREWERMANN, 1972. Voir aussi D.R. DUfOUR, Les mystères de la trinité, Paris, Gallimard, 1990. 
profondeur, transcendance, abîme ou mystère ; mais, sortant de soi, il se dit dans son Logos, la manifestation de son Être caché ; et, retournant à lui-même, il retrouve son unité dans l'Esprit. Dieu est trinité.

Dieu est, en son être même, réciprocité, communion, être-avec, inhabitation mutuelle et dialectique. La tradition chrétienne utilise les symboles de Père, de Fils et d'Esprit, pour dire ce Dieu vivant en luimême et ce Dieu vivant de la création et de l'histoire humaine. Mais ici encore, ces symboles personnalistes doivent être complétés et accompagnés par les concepts analogiques et transpersonnels de l'ontologie : le Père est la manière d'être transcendante de Dieu en luimême (Être en soi) ; le Fils est la manière d'être de Dieu tourné hors de lui-même (Logos révélateur) ; l'Esprit est la manière d'être de Dieu comme présence ramenant tout à l'unité divine (Force unificatrice). La nouvelle physique également peut être récapitulée théologiquement, car elle aussi dévoile un univers un et trine : le cosmos y apparaît dans sa profondeur comme une énergie (Être, Père), intelligible (Logos, Fils) et liante (Amour, Esprit). C'est donc dire que la théologie peut accomplir en quelque sorte les cosmologies ontologique et scientifique et que ces dernières peuvent à leur tour forcer la théologie à expliciter sa dimension cosmologique.

\subsection{La création : la révélation cosmique}

Dans la mesure où la théologie adopte cet horizon cosmologique, elle peut développer une théologie de la création comme grâce originelle, une vision de l'univers comme révélation cosmique et une représentation du cosmos comme lieu de l'inhabitation divine.

D'abord, c'est la totalité de l'être créé qui apparaît comme la première forme de la grâce : la création est bel et bien la grâce originelle ${ }^{35}$. Dieu, l'Être en soi, a créé l'univers, le crée dans le présent, le conduit vers sa fin et l'accomplira dans le futur, dans sa bienveillance. L'univers, se manifestant en son essence comme création de Dieu, s'enracine dans le Fondement divin permanent, qui lutte pour le maintenir dans l'être et triompher du non-être qui l'assaille de l'intérieur de sa

35. Edward H. SCHILLEBEeCKX, Le Christ sacrement de la rencontre de Dieu, Paris, Cerf, 1960, p.256 ; Tillich 1970,(1) p. 237 ; Fox, 1995, (1) p.21, 29, 47, 51, 61 ; SIEGWALT 1996, p. 45 ; HALL, 1998, p.215ss. 
finitude ${ }^{36}$. Ainsi, le cosmos reçoit-il son être comme son pain quotidien : telle est la bénédiction universelle et telle est la grâce originelle ! Alors, c'est le monde en son essence qui est vu comme un don gracieux ou comme un fait de grâce. Ici, il va sans dire, la conception purement surnaturaliste et intimiste de la grâce se trouve dépassée. Qui plus est, Dieu, l'Être en soi, donne à sa création la créativité elle-même qui, par la gratuité et la générosité qui la caractérise, rend à son tour témoignage de la bénédiction universelle et de la grâce originelle ${ }^{37}$.

Ensuite, c'est l'univers qui, depuis sa dimension de profondeur, apparaît comme révélation cosmique de $\mathrm{Dieu}^{38}$. Le cosmos est la révélation première, originelle et universelle ; il est le premier sacrement, le grand livre et «le poème de Dieu » (Augustin) ${ }^{39}$. La Parole de Dieu est en effet en lui comme sagesse cosmique : les philosophes parlent d'une Raison dans l'Être et les physiciens, de l'intelligibilité du cosmos. En son essence, le cosmos est véritablement une musique divine, par delà et à travers la musique des sphères et de l'âme humaine ${ }^{40}$. Lui prêter oreille, c'est déjà entendre les sons de la mélodie divine, en explorer l'intelligibilité ; c'est aussi chercher à comprendre le Logos divin. Car le Logos créateur de Dieu a laissé des traces dans la création; il a planté sa ressemblance en toutes créatures, comme dit Maître Eckhart. Il y a, dans la création, des « logoi spermatikoi », des semences d'intelligibilité, d'ordre et de beauté présentes dans les êtres,

36. Cette vision du Fondement divin permanent dépasse le "déisme ", selon lequel Dieu agit, au commencement, comme premier moteur et semble par la suite absent.

37. Cette créativité et cette gratuité sont largement évoquées dans la nouvelle physique.

38. FOX, 1995,(1) p.37ss ; Guy-Marie BERTRAND, La révélation cosmique Montréal, Bellarmin, 1993, p.158ss ; Tillich, 1970,(3) p.69 ; Gérard SIEGWalt, Dogmatique pour la catholicité évangélique. Tome III. L'affirmation de la foi. 1. Cosmologie théologique: Sciences et philosophie de la nature. Paris, Cerf, 1996, p.85ss ; SiEgWalt, 2000, p.23ss ; MOLTMANN 1988, p.91ss ; Hall 1998, p. 270.

39. Que l'univers soit la première révélation de Dieu, on en trouve le fondement biblique dans des textes comme les suivants : Genèse 1, Psaumes 19, 24, 104, Sagesse 8, 9, 13, Proverbes 1, 8 ,9, Jean 1, Rom.1, Actes des apôtres 17.

40. Eugen Drewermann, De la naissance des dieux à la naissance du Christ. Paris, Seuil, (Points Essais \#355), 1992, p.32. 
et des « vestigia Dei », des signes qui masquent et révèlent à la fois le Logos de Dieu, source de la sagesse immanente du cosmos.

Enfin, le cosmos devient le lieu de l'inhabitation de Dieu ${ }^{41}$. C'est aussi cela que l'on appelle la vision panenthéiste, selon laquelle " tout est en Dieu et Dieu est en tout " : cette conception s'oppose de fait autant à un supranaturalisme dualiste, où Dieu demeure de l'autre côté de sa création, qu'à un panthéisme moniste, où Dieu est identifié à la nature. Ainsi Dieu se contracte-t-il en sa création, en une sorte de "kénose » cosmique. Et c'est par son Esprit qu'il assure sa présence universelle dans le cosmos. L'Esprit du matin de la création, qui planait sur les eaux du chaos originel, est l'Esprit qui inhabite cet univers, le dirigeant vers son accomplissement, dans un incessant combat contre les forces du néant et de la destruction. Il y a une Pentecôte de la création, un Esprit cosmique universel, qui pousse l'univers vers l'actualisation de ses potentialités : l'évolution cosmique révélée dans la nouvelle physique peut être ici sublimée ou reprise théologiquement. De même, peut être récapitulée cette idée de "l'Âme du monde ", dont parlaient déjà Platon, les Stoïciens et Plotin et que Schelling a reprise. Dieu inhabite l'univers et l'Esprit cosmique est l'Âme du monde, tout comme le monde peut être dit « le corps de Dieu ", selon l'expression de Jacob Boëhme, aussi reprise par Panikkar. Cet Esprit qui pousse à l'accomplissement, faut-il ajouter, est également et inséparablement Esprit de communion, créant la solidarité de tous les êtres dans une unique communauté créaturelle, écho du grand tout cosmique dont parlent les physiciens. Et l'homme est aussi solidaire des autres parties de ce tout. Lui également est un être cosmothéandrique : cosmique, divin et humain. Mais "imago Dei », il est peut-être d'abord "imago mundi ", son être étant un " êtreavec » le monde créé. C'est pourquoi, en tant qu'image de Dieu, il doit agir comme un lieutenant de Dieu, solidaire de la nature tout entière, lieu de l'inhabitation divine.

41. Fox, 1995, (1) p.113 ; BERTRAND, 1993, p.555 ; TILliCH 1971, (vol.3) p.107 ; Siegwalt, 2000, p.147, 223 ; Raimundo PANIKKAR, Le culte et l'homme séculier. Paris, Seuil, 1976, p.67, 81, 95 ; Pierre TEILHARD DE Chardin, L'énergie humaine. Paris, Seuil, 1962, p.36; Eugen Drewermann, De l'immortalité des animaux. Paris, Cerf, 1993, p.19, 29 ; HALl 1998, p.10, 27, 192, 268, 281 ; MOLTMANN 1988, p. 8, 23ss, 94ss, 121,131 ss. 
Dieu est donc présent à sa création selon ses trois manières d'être. En tant que Père, il est l'Être en soi avec sa puissance infinie (ontologie) et l'Énergie créatrice de l'univers (nouvelle physique) : et c'est là la grâce originelle. En tant que Fils, il est le Logos universel (ontologie) et l'intelligibilité du cosmos (nouvelle physique) : et c'est là la révélation cosmique. En tant qu'Esprit, il est l'unique Âme du monde animant tous les êtres (ontologie) et la Force de liaison créant le grand tout cosmique (nouvelle physique) : et c'est là l'inhabitation divine.

\subsection{Le Christ : manifestation du Logos universel}

Une représentation du Christ qui, tout en restant fidèle au noyau essentiel de la révélation évangélique, accueille le discours de la cosmologie ontologique et de la cosmologie scientifique, peut insister sur les trois traits suivants : le Christ comme lieu de l'être nouveau, comme manifestation du Logos universel et comme premier-né d'entre les morts.

Le Christ est d'abord celui en qui le don de l'Être s'achève, en quelque sorte : en lui, l'être nouveau apparaît dans l'histoire humaine et dans le devenir cosmique ${ }^{42}$. De la sorte, il est bel et bien porteur du sens de l'évolution cosmique. Car la nouvelle création, vers laquelle chemine l'univers entier, se révèle en lui. Le nouvel être réside ainsi dans une victoire, en premier lieu : c'est celle du triomphe du Christ sur les conditions existentielles de souffrance, d'aliénation et de mort, avec leurs conséquences destructrices; s'y étant soumis jusqu'à la mort en croix, il en est sorti victorieux dans la résurrection. Dans sa "kénose ", dans son abaissement et dans son dépouillement les plus profonds, a surgi l'être nouveau, victorieux du tragique de l'existence. Cette victoire de l'Être sur le non-être, telle est la grâce du nouvel être manifesté dans le Christ. Mais le nouvel être apparaît également comme une profonde énergie de réconciliation : le Christ est l'être cosmothéandrique par excellence, en qui sont unis en profondeur l'être humain, Dieu et le cosmos ; il est l'être relié à son Fondement divin, qui dit ce qu'est la création telle que Dieu la souhaite, c'est-àdire un tout unifié dans lequel l'amour gracieux de Dieu relie et habite toutes les créatures et s'avère ultimement plus fort que la mort.

42. Maldamé, 1993, p. 136, 162 ; Tillich, 1970, (3) p. 43ss ; Tillich 1971, (vol.3) p.12, 90ss, 14ss ; Fox, 1995, (2) p. 25, 120, 231. Sur ce thème, voir par exemple Gal.6,15 ; Rom.6,8 ; Col.1,15-20. 
Le Christ est aussi une manifestation du Logos divin et cosmique $^{43}$. Le Logos divin est le principe de la manifestation de Dieu en lui-même et dans sa création. Il est l'image du Dieu invisible et transcendant. Il apparaît dans la création comme la sagesse cosmique universelle, dont parlent la Bible, la cosmologie ontologique et la nouvelle physique elle-même. La puissance et la lumière du Logos " irradient dans la matière ", selon l'expression de Teilhard de Chardin. C'est le Logos divin et cosmique qui se révèle de façon particulière dans le Christ. En Jésus le Christ, il y a événement-avènement, « kairos », manifestation et sacrement du Logos universel et éternel pour les êtres humains. Ce Logos qui est avec Dieu, qui est le Fils de Dieu, par qui tout a été fait, qui de tout être est la vie et qui se manifeste de façon unique dans une chair humaine, celle de Jésus le Christ, parle ultimement de la filialité divine et de la sagesse cosmique présentes en l'essence même de toutes les créatures.

Enfin, le Christ est premier-né de toute créature, premier-né d'entre les morts et aîné d'une multitude de frères ${ }^{44}$. En ce sens, il est le prototype de la création telle que Dieu la souhaite, le symbole archétypique universel révélant l'essence divine cachée ou la « christicité » de l'humanité et de la création tout entière. Son abaissement et sa glorification ont une dimension cosmique. En son baptême dans l'Esprit, en sa transfiguration, en sa mort-résurrection, il rassemble le destin de l'univers. Il est le Christ cosmique, le "Pantocrator ", celui qui récapitule en son être et en son action la dignité de toute créature. Il est par excellence le microcosme, l'être cosmothéandrique, à la fois résumé de la création et prémices de son accomplissement. À ce titre, il est le premier-né dans l'Esprit, le Nouvel Adam, témoignant de la recréation ou de l'achèvement gracieux du monde dans l'Esprit. Sa souffrance et son élévation ont une ampleur cosmique.

43. Maldamé, 1993, p.183; Pierre Teilhard de Chardin, Le milieu divin. Paris, Seuil, 1957, pp.49, 55, 147 ; TILliCH, 1970,(3) pp. 43, 69, 71 ; Tillich, 1971, (vol.2) pp.15, 95, 149, 164, 167 ; Fox, 1995,(1) p. 112 ; Fox, 1995,(2) p.312 ; SIEGWALT, 2000, p.206ss. Voir notamment Jean 1, 1-15 et Heb. 1,1-2.

44. 1 Cor.15, 27-28 ; Rom. 8, 29 ; Eph. 1, 3-23 ; Col.1, 15-20 ; Phil. 2, 5 ss ; Jean 15, 5 . 
Ainsi, le Père accomplit dans le Christ le don de l'être, le Fils se manifeste comme Logos en lui et l'Esprit qui l'a ressuscité répand cette grâce en toute la création.

\subsection{L'Église : la communauté de l'Esprit}

Une théologie ouverte à sa dimension cosmologique présentera les communautés ecclésiales comme reliées à l'Esprit présent dans le Christ, certes, mais en insistant sur le fait qu'il s'agit de l'Esprit inhabitant la création entière. Dans cette optique, on peut dégager trois traits de ce qu'on peut appeler "l'essence invisible » des communautés ecclésiales concrètes toujours engagées, pour leur part, dans les contradictions et les ambiguités de l'existence.

Premier trait : la communauté spirituelle ${ }^{45}$. Assurément, c'est l'Esprit présent dans le Christ qui est à l'œuvre dans les communautés ecclésiales et dans l'histoire humaine. C'est cet Esprit de la Pentecôte, Esprit de la nouvelle naissance, de la régénération et de la participation à l'être nouveau, qui agit dans la communauté spirituelle. Ainsi les communautés ecclésiales sont-elles, à travers les contradictions et les ambiguïtés de la vie, des témoins de ce travail de l'Esprit cherchant à réaliser l'être nouveau. Mais la théologie cosmologique ajoutera que cet Esprit présent dans le Christ est le même que l'Esprit inhabitant la création; le même que cette puissance infinie de l'Être en soi en lutte permanente contre le non-être dans la totalité de l'être, lutte dont parle la cosmologie ontologique ; le même que cette Force spirituelle poursuivant le combat pour l'unité de tout le cosmos, combat évoqué dans la nouvelle physique. L'Esprit régénérateur, construisant la communauté spirituelle, est l'Esprit créateur, poursuivant l'établissement de l'unique communauté des créatures.

Deuxième trait : la communauté théologale ${ }^{46}$. Les fruits de l'Esprit ou les marques de l'être nouveau sont la foi, l'espérance (dont nous

45. Joseph Fromaget, L'homme tridimensionnel. Paris, Albin Michel, 1996, passim ; Tillich, 1971, (vol.2) p. 176ss ; Ibid. 1971, (vol.3) pp. 138ss, 155ss, 162, 178 ; SIEGWALt, 2000, p. 220 ; MOLTMANN, 1988, p.25.

46. Fromaget, 1996, passim; Tillich 1970, (3) p.51ss ; Paul Tillich, Dynamics of Faith. New York, Harper and Row, 1958, passim ; Ibid. 1971, (vol.2) p.158ss ; Alain BADIOU, Saint Paul, La fondation de l'universalisme. Paris, PUF, 1997, p.91 ; PANNIKKAR, 1976, p. 22. Voir Gal. 5, 22. 
parlerons plus loin) et l'amour. La communauté spirituelle rassemble en effet les personnes dont l'existence est ouverte à la transcendance de l'Être : leur souci ultime, leur préoccupation inconditionnelle, leur passion de l'Absolu et, pour tout dire, leur foi réside dans le fait d'être saisi par la puissance de l'Esprit, source du nouvel être révélé dans le Christ. Ces personnes acceptent donc, dans la foi, d'être portées et transformées par l'Esprit présent dans le Christ, ce même Esprit qui inhabite la création tout entière. Elles acceptent aussi d'accueillir le Fondement de leur être comme "agapè » ou amour universel, inconditionnel et désintéressé, amour qui constitue l'essence de toute vertu humaine, la norme ultime de tout contenu éthique et l'accomplissement même de la foi. Car l'Esprit présent et révélé dans le Christ, l'Esprit de la création et de la régénération, est l'Esprit de communion, sans nul doute au cœur de cet élan vers l'unité originelle dont parle la cosmologie ontologique et de cette force de liaison créant la totalité cosmique dont parle la nouvelle physique.

Troisième trait : la communauté œcuménique ${ }^{47}$. Il y va ici, à l'encontre de toute emprise communautaire et de vision restreinte de l'Église ou du peuple élu, de l'universalisme de la communauté spirituelle, qui inclut tous ces hommes et toutes ces femmes "de bonne volonté ", dont le contenu du souci ultime vise, à travers les combats ambigus de la vie, l'amour inconditionnel, la justice pour tous et le respect de toute créature. Eux aussi, artisans de la beauté du monde, malgré les contradictions de leur vie, portent les marques de l'être nouveau et témoignent des fruits de l'Esprit. Ils sont, comme dit Tillich, «la communauté spirituelle dans sa latence séculière ${ }^{48}$ ». Mais l'universalisme de la communauté spirituelle va encore plus loin : il inclut la solidarité et l'interdépendance cosmique de toutes les créatures. C'est en ce sens que les membres (explicites ou implicites) de la communauté spirituelle sont appelés à contribuer à la naissance continue du cosmos et à participer ainsi à la cosmogenèse, à devenir intendants ou coopérateurs de Dieu et à témoigner de la sorte de leur être-avec-la-nature, à se faire artisans de la beauté du monde, puisque l'Esprit de la création révélé et présent dans le Christ est Esprit de communion.

47. Fox, 1995, (1) pp.21, 118, 125, 226ss, 275 ; Moltmann, 1988, pp.18, 25 ; Alain BADIOU, Saint Paul, La fondation de l'universalisme. Paris, PUF, 1997, pp.6, 15, 23, 61 ; HALL, 1998, pp.207, 256, 292-293.

48. Tillich, 1971, (vol.3) p.153. 


\subsection{La liturgie : la célébration cosmique}

La liturgie, retrouvant elle aussi ses sources, peut devenir, si elle ne l'est déjà, une authentique célébration cosmique, selon trois dimensions. Dans une première dimension ${ }^{49}$, elle rappelle que le cosmos luimême célèbre Dieu. Chaque créature, actualisant son essence en dépit des contradictions de l'existence, chante en effet la gloire de Dieu. En ce sens, il existe une première liturgie cosmique, une eucharistie primordiale de la création tout entière. Toutes les créatures du cosmos célèbrent Dieu par leur être profond et par leur joie d'exister (ce sont des êtres eucharistiques), tout comme elles le prient d'ailleurs, puisqu'elles gémissent aussi dans les souffrances de leur enfantement et de leur mort (ce sont des orantes). Mais il y a plus encore. La liturgie ecclésiale utilise en effet des éléments cosmiques, tels le feu ou l'eau. Elle les révèle dans leur dimension de transcendance; elle en montre le caractère symbolique et archétypique; elle en fait des sacrements, en quelque sorte. En eux, le sensible devient explicitement le témoin de l'invisible : en cela réside d'ailleurs leur profonde beauté.

C'est cette profonde beauté cosmique que la communauté ecclésiale doit aussi célébrer dans sa liturgie ${ }^{50}$. Un culte à dimension cosmologique remercie les êtres d'exister; il bénit la vie ; il fête cet univers jailli du chaos; il exprime sa gratitude au temps et à l'espace de la cosmogenèse. Puis, le culte célèbre en même temps notre appartenance à ce cosmos; il reconnaît que notre être est un pur don de l'Être, voire une grâce particulière inséparablement liée à cette grâce originelle qu'est la création tout entière ; et il prie également pour la création souffrante, invitant tous les hommes à contribuer au recul des frontières du mal sur leur petite planète et à se faire eux-mêmes, dans un véritable combat spirituel contre les forces de destruction, artisans de la beauté du monde.

Enfin, dans sa liturgie, l'Église est aussi appelée à célébrer le Dieu créateur. En ce sens, la liturgie devient une réponse à la beauté essentielle de la création de Dieu ; elle rend grâce pour cette grâce universelle de Dieu qu'est la création. Dans le culte ecclésial, c'est donc aussi l'être

49. Maldamé, 1993, p.247ss ; FoX, 1995, (1) p.120 ; DreWERMANN, 1992, p.54ss ; SIEGWALT, 2000, pp.108, 114 ; MOLTMANN, 1988, p.100-101. 50. Fox 1995, (1) passim ; MOLTMANN 1988, pp.108, 144, 363. 
humain qui exprime, en ses gestes et ses paroles, la louange cosmique ; c'est lui qui célèbre la gloire de Dieu déjà présente dans toute la création. En tant qu' " imago mundi ", l'être humain est lui aussi un microcosme ou un résumé de l'univers et il représente alors la création auprès de Dieu, tout comme en tant qu' "imago Dei ", il représente Dieu auprès de sa création. Lorsque la liturgie cosmique est ainsi chantée par l'homme, c'est toute la création qui parle à Dieu par la voix humaine.

\subsection{L'espérance : la nouvelle création}

Dans l'optique d'une théologie affirmant sa dimension cosmologique, on peut faire une "relecture cosmique », dépassant par le fait même une interprétation purement anthropologique et individualiste, de trois symboles complémentaires de l'espérance : la résurrection, la participation à la vie éternelle et la nouvelle création. Ces symboles de l'espérance, faut-il le rappeler, sont soumis à la dialectique chrétienne du « déjà-là » (de la préfiguration et de l'anticipation), et du "pas encore »(de l'accomplissement et de la plénitude).

Le symbole de la résurrection se rattache directement à la résurrection du Christ, événement à la fois unique et symbolique, qui témoigne de la transfiguration de toutes les dimensions de l'existence au-delà de la mort ${ }^{51}$. Cette façon de nommer l'espérance montre que la matérialité possède une profondeur spirituelle qui, incluse dans la mouvance de l'Esprit créateur et régénérateur, peut transcender la mort : l'apôtre Paul parle d'un " corps spirituel ». La matière cosmique, dont fait partie la matérialité humaine, est une matière subtile, disent les physiciens; dans le holomouvement d'impliement et de dépliement, de destruction et de création, de mort et de résurrection, elle peut être transformée en énergie pure. Ainsi, la vieille réalité transfigurée surgit de la mort et apparaît comme être nouveau. Et la résurrection du Christ, prototype de toute résurrection, n'est-elle pas la " récapitulation » de ce désir de toute existence matérielle, à commencer par celle de l'homme, de surgir de la mort et de s'achever comme énergie et matière spirituelle ? N'annonce-t-elle pas l'entrée de l'uni-

51. MoltmanN, 1988, p. 96 ; Tillich, 1970, (1) p. 225 ; Tillich, 1971, (vol.3) p. 414 ; DREWERMANN, 1992, p. 201 ; DREWERMANN, 1993, p. 17ss. 
vers entier dans un autre niveau d'être, où la lumière et la vie triomphent des ténèbres et de la mort ?

La participation à la vie éternelle est un autre symbole chrétien de l'espérance historique et eschatologique ${ }^{52}$. Cette vie éternelle, révélée dans le Christ comme " déjà-là » et " pas-encore » accomplis en tout être, est un espace-temps autre, intégré dans l'éternité divine, et non un simple espace-temps sans fin. Lorsque l'éternel fait ainsi irruption dans l'espace-temps, c'est la dimension de transcendance de ce dernier qui apparaît : il y a " kairos », moment de grâce, moment d'éternité. Alors le sens de tout espace-temps se révèle : surmonter la rupture entre l'essence et l'existence, vaincre la séparation entre le potentiel et l'actuel, en chaque être, dans l'histoire humaine et dans le cosmos tout entier. C'est ce que Schelling appelait "l'essentialisation ", concept réinterprété par Tillich et Siegwalt. Dans ce mouvement d'essentialisation universelle, l'essence théonome d'un être tout comme celle de la création entière, c'est-à-dire son ouverture à la transcendance et son être-avec-Dieu, s'accomplit dans les conditions contradictoires et ambiguës de l'existence concrète. Alors, l'espace-temps humain et cosmique retrouve son Fondement et l'Esprit créateur et régénérateur se manifeste en lui, l'intégrant à la vie éternelle. L'espace-temps entre dans l'éternité ; l'éternité apparaît dans l'espace-temps. Telle était la grâce de l'Esprit manifestée dans le Christ et telle est la grâce de l'Esprit promise à la création. La participation à la vie éternelle apparaît ultimement comme la fin intrinsèque non seulement de chaque personne et de toute l'histoire humaine, mais aussi comme le désir et l'effort d'accomplissement qui traversent en profondeur l'évolution cosmique.

Et c'est à ce point précis qu'entre en jeu un autre symbole de l'espérance chrétienne : l'avènement de la nouvelle création ${ }^{53}$. Elle aussi, elle s'est déjà révélée dans le Christ. Mais c'est toute la création qui gémit dans les douleurs de l'enfantement ; la gestation de la nouvelle création est en elle, le levain de l'être nouveau est en elle, et cette

52. SiegWalt, 2000, pp.133, 214, 381, 467 ; Hall, 1998, p.238 ; Tillich, 1971, (vol.3) pp. 358, 373, 394, 398ss, 414ss. Tout l'évangile de Jean est imprégné de ce symbole de la vie éternelle.

53. MALDAMÉ, 1993, pp. 223ss, 271 ; Tillich, 1971, (vol.3 ) pp. 405, 421 ; FoX, 1995, (2) p. 229 ; SIEGWALT, 2000, pp.53, 95, 117, 191, 418, 442, 464 ; Moltmann, 1988, p.17ss. Voir 1 Cor.15, 18-28 ; Col.3, 11 ; 
gestation et ce levain sont repris par l'Esprit. Si le désir d'accomplissement caractérise la création, si la quête de la complétude l'habite en profondeur et si l'ouverture à un nouvel état d'être existe en elle (l'évolution cosmique ne témoigne-t-elle pas de ce mouvement ?), c'est pourtant l'Esprit cosmique et divin, créateur et régénérateur, qui produit le dépassement souhaité, qui donne en toute grâce la plénitude de l'être. C'est lui qui, Âme du monde, le régénère et le renouvelle de l'intérieur, pour en faire la demeure de Dieu. Et l'histoire humaine, et l'évolution cosmique accomplies aux temps eschatologiques, c'est Dieu se manifestant dans un monde réconcilié avec son être profond et théonome. C'est aussi "Dieu en tout et tout en Dieu ", comme le rappelle la formule panenthéiste.

$$
* * *
$$

Ainsi, puisant à ses sources profondes, la théologie chrétienne peut s'ouvrir de façon critique à la nouvelle physique et à la cosmologie ontologique : elle contribue alors à les mener au dépassement dans la vérité de leur démarche, tout en explicitant elle-même ses propres contenus dans la vérité de son cheminement. C'est là une dimension essentielle du combat spirituel qu'elle doit aujourd'hui mener : un combat pour l'universalisme, comme celui de l'apôtre Paul, où il n'y a plus ni Juif ni Grec, ni homme ni femme, ni soleil ni terre. Il y a seulement le cosmos que Dieu, par son Logos et dans son Esprit, a créé, crée continuellement et recréera en l'accomplissant en son essence, à l'image du Christ : toujours comme une grâce et un don gratuit de l'être.

\section{RÉSUMÉ}

Cet article montre qu'un aspect essentiel du combat spirituel de la théologie chrétienne, aujourd'hui, est de retrouver sa dimension cosmologique. Puisant à ses propres sources, la théologie peut accueillir de façon critique la cosmologie scientifique contemporaine (la « nouvelle physique ») et ses prolongements et enracinements ontologiques.

"Récapitulant» ainsi science et philosophie, la théologie les conduit vers leur propre dépassement ; et, grâce à elles, elle chemine elle-même vers sa vérité la plus riche, puisqu'elle est amenée à relire les représentations et les contenus essentiels de la foi : Dieu, la création, le Christ, l’Église, la liturgie et l'espérance. 


\begin{abstract}
This article demonstrates one of the most important aspects of spiritual combat in Christian theology today - the rediscovery of its cosmic dimension. Drawing from its own sources, theology can, in a critical fashion, embrace the contemporary scientific cosmology (the new physics) and its ontological roots and extensions.

By "fusing" science and philosophy, theology can encourage these disciplines to move beyond their own limitations, and in the process be enriched by a reassessment of its own representation of the contents of faith : God, creation, Christ, the Church, liturgy and hope.
\end{abstract}

